OPEN ACCESS

Edited by:

Junji Xing,

Houston Methodist Research Institute,

United States

Reviewed by:

Björn Rissiek,

University Medical Center

Hamburg-Eppendorf, Germany

Kobra-Omidfar

Tehran University of Medical

Sciences, Iran

*Correspondence:

Yurong Wen

Yurong.Wen@xitu.edu.cn

Steve Schoonooghe

steve.schoonooghe@vub.be

Geert Raes

Geert.Raes@vub.be

tThese authors share first authorship

¥These authors share senior authorship

Specialty section

This article was submitted to

Molecular Innate Immunity,

a section of the journal

Frontiers in Immunology

Received: 16 December 2020 Accepted: 01 February 2021

Published: 22 February 2021

Citation:

Zheng F, Zhou J, Ouyang Z, Zhang J,

Wang $X$, Muyldermans $S$, Van Ginderachter J, Devoogdt N, Wen Y, Schoonooghe $S$ and Raes G (2021)

Development and Characterization of Nanobodies Targeting the Kupffer Cell. Front. Immunol. 12:641819. doi: 10.3389/fimmu.2021.641819

\section{Development and Characterization of Nanobodies Targeting the Kupffer Cell}

\author{
Fang Zheng ${ }^{1,2,3 \dagger}$, Jinhong Zhou ${ }^{1,4+}$, Zhenlin Ouyang ${ }^{4}$, Jiaxin Zhang ${ }^{1}$, Xinyi Wang ${ }^{1}$, \\ Serge Muyldermans ${ }^{2}$, Jo Van Ginderachter ${ }^{2,3}$, Nick Devoogdt ${ }^{5}$, Yurong Wen ${ }^{1,4 *}$, \\ Steve Schoonooghe ${ }^{2,3 * \pm}$ and Geert Raes ${ }^{2,3 * \neq}$ \\ ${ }^{1}$ The Key Laboratory of Environment and Genes Related to Disease of Ministry of Education, Health Science Center, Xi'an \\ Jiaotong University, Xi'an, China, ${ }^{2}$ Research Group of Cellular and Molecular Immunology, Vrije Universiteit Brussel, Brussels, \\ Belgium, ${ }^{3}$ Laboratory of Myeloid Cell Immunology, VIB Center for Inflammation Research, Brussels, Belgium, ${ }^{4}$ Talent \\ Highland, The First Affiliated Hospital, Xi'an Jiaotong University, Xi'an, China, ${ }^{5}$ In vivo Cellular and Molecular Imaging \\ Laboratory, Vrije Universiteit Brussel, Brussels, Belgium
}

Nanobodies that are derived from single-chain antibodies of camelids have served as powerful tools in diagnostics, therapeutics and investigation of membrane receptors' structure and function. In this study, we developed a series of nanobodies by a phage display screening building from lymphocytes isolated from an alpaca immunized with recombinant mouse Kupffer cell receptor Clec4F, which is involved in pathogen recognition by binding to galactose and $\mathrm{N}$-acetylgalactosamine. Bio-panning selections retrieved 14 different nanobodies against Clec4F with an affinity ranging from 0.2 to $2 \mathrm{nM}$ as determined by SPR. Those nanobodies mainly recognize 4 different epitopes as analyzed via competitive epitope binning. By analysis of the radioactivity in each organ after injection of ${ }^{99 \mathrm{~m}} \mathrm{Tc}$ labeled Clec4F nanobodies in naïve mice, we found that these nanobodies are targeting the liver. Furthermore, we performed a structural characterization at atomic resolution of two of the Clec4F nanobodies from different epitope groups, which revealed distinct features within the CDR2 and CDR3 regions. Taken together, we developed a series of nanobodies targeting multiple distinct recognition epitopes of the Kupffer cell-specific receptor Clec4F which may be useful for its structural and functional investigation as well as for use as molecular imaging and therapeutic agents.

Keywords: Clec4F, nanobody, epitope binning, x-ray crystallography, Kupffer cells

\section{INTRODUCTION}

Macrophages seem to adapt to different tissues with specific functions during development and adulthood, hence the tissue-resident macrophages are extremely heterogeneous (1). The identification of tissue-resident macrophages and their unique feature in relation to other immune cells has so far mainly relied upon the detection of their anatomical position $(2,3)$. Apart from the heterogeneity observed between different organs, even within the same tissues, there appear to be subpopulations of specialized macrophages that exhibit unique characteristics (4). Physically, Kupffer cells (KCs) are interspersed with fenestrated liver sinusoidal endothelial cells in a mosaic fashion to make up the sinusoidal lining. KCs express several cell-surface receptors and receptor 
complexes involved in immune stimulation (5). These include complement receptors (CRs), Fc-receptors, C type lectins (CLR), which are capable of directly binding microbial surface components such as sugars and polyanionic moieties, adhesion receptors, and receptors for polysaccharides of microbial and host origin (6-8). Targeting of markers expressed on KCs and inflamed epithelium offers perspectives for monitoring the inflammation development and understanding the type of specific cells and receptors involved (7). The most frequently used markers for KCs are CD68, CD11b, and F4/80 in mouse. The presence of $\mathrm{F} 4 / 80$ antigen (which becomes expressed as monocytes mature into tissue macrophages) on sinusoidal liver cells has been used to "define" KCs (9).

Recently, we have identified $\mathrm{Clec} 4 \mathrm{~F}$ as resident liver macrophage marker. Clec4F (C-type lectin domain family 4, member F), is a type II transmembrane glycoprotein member of the C-type lectin superfamily (10). Clec4F can bind galactose and $\mathrm{N}$-acetylgalactosamine. The mouse $\mathrm{Clec} 4 \mathrm{~F}$ forms a trimeric coiled-coil interface within its heptad neck and constitutes conserved $\mathrm{Ca}^{2+}$-dependent carbohydrate-recognition domains, however as compared to other C-type lectin family members, Clec4F exhibits a distinct distance between the $\mathrm{Ca}^{2+}$ binding site within the trimer which is proposed to enable binding of specific glycans chain (11). Humans cannot encode a functional Clec4F receptor due to a mutation in the splice acceptor site of the last exon preventing appropriate splicing, and a missense mutation disrupting the sugar-binding site (12). Although Clec $4 \mathrm{~F}$ is present in rodents but not in humans, Clec4F is starting to be used as a marker in immunity and in vivo imaging in mouse disease models to study $\mathrm{KCs}^{11}$. Based on Clec4F, together with Tim4, Decisscher et al. were able to distinguish between monocyte-derived macrophages $(\mathrm{MoM} \varphi s)$ and KCs in the Non-alcoholic steatohepatitis (NASH) liver $(10,13)$. Coupling of the diphtheria toxin receptor to the Clec4F promoter (KC-DTR mice) allowed the specific ablation of KCs and KC-DTR mice, providing a powerful tool for ZEB2 identification (14) as a KC-specific transcription factor. Clec4F-deficient mice produced far less cytokines than wild type littermates after intravenous injection of $\alpha$-GalCer, which binds to CD1d and is a typical stimulator of active NKT cells (15), which suggests that Clec4F may be involved in presentation of glycolipid antigens or $\alpha$-GalCer to NKT cells and thus could be an important ligand to activate liver KCs.

Molecular engineering of the heavy-chain antibodies' antigenbinding domains created the VHHs, having unique biophysical properties that render them attractive for biotechnological applications. The name nanobody $(\mathrm{Nb})$ was coined to refer to the recombinantly-produced form of the VHH (16). As compared to other antibody fragments consisting of multiple domains, the $\mathrm{Nb}$ domains have a characteristic prolate ellipsoid shape that allows them to target cavities deep within their target antigens, therefore, $\mathrm{Nb}$ domains are frequently observed to target hidden or conformational target epitopes, which offers an added value for many applications (17). Owing to their unique properties, $\mathrm{Nb}$ domains have become increasingly popular for purposes of diagnostic or therapeutic applications in various disease areas, including infectious, inflammatory and oncologic diseases $(18,19)$.

Previously, we reported the construction of an immune phage display library for retrieving Clec $4 \mathrm{~F}$ nanobodies. Furthermore, a lead Clec4F nanobody, $\mathrm{Nb} 2.22$, was identified to bind to KCs in FACS and in immunohistochemistry (20). It was also shown there that the ${ }^{99 \mathrm{~m}} \mathrm{Tc}-\mathrm{Nb} 2.22$ signal in liver upon in vivo imaging disappears after chlodronate liposome depletion of KCs, demonstrating that the in vivo signal in the liver is restricted to KCs (10). In the current manuscript, we provide more details on how we immunized an alpaca against Clec4F and isolated a number of Clec4F nanobodies that display high affinity. We demonstrated by epitope binning that these 25 Clec4F nanobodies mainly bind to 4 individual epitopes of Clec4F. The protein structure of Nb2.22 clearly reveals enhanced flexibility in the CDR3 as compared to Nb1.46. The series of nanobodies targeting multiple distinct epitopes of Kupffer cell receptor Clec4F may be beneficial for its structural and functional investigation as well as for future use in molecular imaging and therapeutic agents.

\section{MATERIALS AND METHODS}

\section{Production of Recombinant Mouse Clec4F}

NS0-derived mouse CLEC4F/CLECSF13 protein, with an Nterminal 9-His tag was ordered from R\&D Systems which the soluble extracellular domain Ala65-Gly548 of Clec4F was expressed in mouse myeloma cell line, and used for alpaca immunization, panning and ELISA screening, largely as described previously (21). VHH phage-display library was generated by RT-PCR on mRNA of peripheral blood lymphocytes isolated from an immunized alpaca (Vicugna pacos) and cloning in the vector pHen4. The repertoire of the VHH library was expressed on phages and panned against microplates coated with Clec $4 \mathrm{~F}$ antigen at a concentration of $10 \mu \mathrm{g} / \mu \mathrm{l}$. After four rounds of selection against recombinant mouse Clec $4 \mathrm{~F}$, individual colonies were picked from round 2 and round 3 of panning, and their VHH was expressed as soluble periplasmic protein.

\section{Enrichment Phage ELISA}

Enrichment phage ELISA was performed for four rounds of bio-panning of phage-displayed Clec4F nanobodies. The antigen containing wells were coated with either $1 \mu \mathrm{g} / \mathrm{ml}$ mouse Clec $4 \mathrm{~F}$ protein, blank wells were coated with PBS. Output phages before panning (round 0) and after 4 rounds of bio-panning were used as ligands in an ELISA. Color was developed using mouse antiM13 antibody followed by anti-mouse AP and AP substrate. The recombinant $\mathrm{VHH}$ containing periplasm was extracted and was tested for antigen recognition in phage enrichment ELISA and the clones which generated a positive signal on Clec $4 \mathrm{~F}$ protein (OD450 nm signal of each clone was divided by signal of well without antigen and considered positive if the resulting ratio was $\geq 3$ ) were chosen for DNA sequencing.

\section{Nanobody Production and Purification}

The Clec4F-specific nanobody gene sequences were inserted into the pHEN6c plasmid and transfected into E. coli WK6 host 
cells. Hexahistidine-tagged (His-tag) Clec4F nanobodies were extracted from E. coli WK6 periplasmic space by osmotic shock. All the extracted proteins were expressed in $400 \mathrm{~mL}$ medium and purified by immobilized metal affinity chromatography (IMAC) using the Ni-NTA column and the buffer was exchanged from $0.5 \mathrm{M}$ imidazole elution buffer to PBS by dialysis. The purity and size of the nanobody was detected by SDS-PAGE and Coomassie brilliant blue staining.

\section{Affinity Evaluation and Epitope Mapping of Clec4F Nanobodies}

After purification, the concentration of Clec4F nanobodies was determined by the Nanodrop spectrophotometer using the theoretical extinction coefficient of the nanobody calculated from its amino acid sequence (7). All selected nanobodies bound to Clec4F antigen with high affinities in $\mathrm{nM}$ range as determined by SPR. Nb affinity experiments were carried out by surface plasmon resonance (SPR, Biacore T200, GE Healthcare). The Clec4F nanobodies were flown at $30 \mu \mathrm{l} / \mathrm{min}$, at different concentrations (1-400 nM) over $400 \mathrm{RU}$ (resonance units) of recombinant mouse Clec4F protein coupled to a CM5 chip. The affinity constants were determined using a 1:1 binding model using the BIACORE evaluation T200 software (GE Healthcare).

Epitope mapping of $\mathrm{Clec} 4 \mathrm{~F}$ nanobodies was also investigated by SPR. Because Nb 1.46, 12.75, and 5.10 were very difficult to elute and regenerate from the Clec4F coated CM5 chip, we excluded them from the epitope binning. Briefly, after a 1st $\mathrm{Nb}$ was injected and reached its saturation plateau, a mix of the 1st $\mathrm{Nb}$ and a $2 \mathrm{nd} \mathrm{Nb}$ was injected to evaluate whether the $2 \mathrm{nd} \mathrm{Nb}$ caused extra binding RU. Next, the sensorgram for each $\mathrm{Nb}$ pair was drawn to investigate the competition profiles.

\section{ELISA}

Clec4F $(1 \mu \mathrm{g} / \mathrm{mL})$ was coated directly on 96-well plates, overnight at $4^{\circ} \mathrm{C}$. Free protein binding sites were blocked by $4 \%$ skimmed milk in PBS for $2 \mathrm{~h}$ at room temperature. Next, a series dilution of Clec4F nanobodies in $100 \mu \mathrm{L} 1 \%$ skimmed milk PBST were added. Detection of antigen bound $\mathrm{Nb}$ was performed by anti-His-tag and subsequently by anti-mouse IgG- alkaline phosphatase antibody. The OD405 nm values of each well were subtracted with the blank (signal from well without Clec4F).

\section{9mTc-Nanobody Labeling and Pinhole SPECT/ $\mu$ CT Analysis}

Based on the yield and purity of the nanobodies, one $\mathrm{Nb}$ representative for each group was selected for further analysis. Nanobodies were labeled with ${ }^{99 \mathrm{~m}}$ Technetium $\left({ }^{99 \mathrm{~m}} \mathrm{Tc}\right)$ via their His-tag through tricarbonyl chemistry as described before (22, 23). Hereby, the $\left({ }^{99 \mathrm{~m}} \mathrm{Tc}\right)$ tricarbonyl precursor was prepared in accordance to the IsoLinkTM kit (Covidien, St. Louis, USA). The kit reduces and carbonylates $\left[{ }^{99} \mathrm{~m} \mathrm{Tc}\right] \mathrm{TcO}_{4}$ into $\left(\left[{ }^{99 \mathrm{~m}} \mathrm{Tc}\right](\mathrm{CO})_{3}\left(\mathrm{H}_{2} \mathrm{O}\right)_{3}\right)^{+}$after heating $\left(100^{\circ} \mathrm{C}\right.$ for $\left.20 \mathrm{~min}\right)$. This precursor $(500 \mu \mathrm{l})$ was next incubated at $50^{\circ} \mathrm{C}$ for $1 \mathrm{~h}$ with $50 \mu \mathrm{g}$ of the His-tagged $\mathrm{Nb}$. The $\left[{ }^{99 \mathrm{~m}} \mathrm{Tc}\right.$ ] tricarbonyl precursor forms a tridentate coordinated complex with every other histidine residue in the hexahistidine complex. The ${ }^{99 \mathrm{~m}} \mathrm{Tc}$-labeled $\mathrm{Nb}$ $\left({ }^{99 \mathrm{~m}} \mathrm{Tc}-\mathrm{Nb}\right)$ solution was subsequently purified on a NAP5 column (GE Healthcare), and passed through a MillexGV4 $0.22-\mathrm{mm}$ filter (Millipore). After ${ }^{99 \mathrm{~m}}$ Tc-radiolabeling and subsequent purification steps, labeling efficiencies, reflecting the amount of the added ${ }^{99 \mathrm{~m}} \mathrm{Tc}$ that ended up coupled to the filtered nanobodies, ranged between 50 and $70 \%$ for the various nanobody preparations. Radiochemical purities, reflecting non-free radioactivity coupled to the nanobody in the final filtered preparation, were determined by instant thin-layer chromatography using acetone as mobile phase and were at least $99 \%$ for all nanobodies. Mice were injected intravenously with $80-100 \mu \mathrm{l}$ of ${ }^{99 \mathrm{~m}} \mathrm{Tc}-\mathrm{Nbs}$, corresponding to $52.79 \pm 19.63$ $\mathrm{MBq}$ (3 mice \pm SEM) per mouse. At $1 \mathrm{~h}$ post-injection, mice were sacrificed, tissues were dissected and weighed, and their radioactivity content was measured using an automated $\gamma$ counter (Cobra II Inspector 5003; Canberra-Packard, Schwadorf, Austria). Organ uptake was calculated as the percentage of injected radioactivity per gram (\%IA/g) and corrected for decay (23). All applicable institutional and/or national guidelines for the care and use of animals were followed and all animal experiments were approved by the Ethical Committee for Animal Experiments of the Vrije Universiteit Brussel (Lab Accreditation Number: LA1210220).

\section{Crystallization and Data Collection}

All crystallization trials were carried out with the sittingdrop vapor-diffusion approach at room temperature. Clec4F nanobodies were purified by size exclusion chromatography on columns pre-equilibrated with $20 \mathrm{mM}$ Tris $\mathrm{pH} 7.5,150 \mathrm{mM}$ $\mathrm{NaCl}$ and 5\% Glycerol. The fractions containing the nanobodies were pooled and concentrated to around $15 \mathrm{mg} / \mathrm{mL}$. The crystallization trials were further carried out by mixing $0.5 \mu \mathrm{L}$ protein complex with an equal volume of reservoir solution. The Nb2.46 was crystallized in $0.02 \mathrm{M}$ Magnesium chloride hexahydrate, 0.1 M HEPES, pH 7.5, 22\% poly acrylic acid sodium salt 5100 and the $\mathrm{Nb} 2.22$ crystals were observed in $0.05 \mathrm{M}$ Citric acid, 0.04 M BIS-TRIS propane $\mathrm{pH} 5.0,16 \%$ polyethylene glycol 3,350 respectively. All the crystals were harvested from the crystallization drop and flash frozen with a cryostream. Single crystals data were collected in the Shanghai Synchrotron Radiation Facility (SSRF) BL18U1 and BL19U1 at a wavelength of $0.98 \AA$ at $100 \mathrm{~K}$. The Nb1.46 crystal diffracted to $1.98 \AA$ with $\alpha$ $=\beta=\gamma=90^{\circ}, \mathrm{a}=\mathrm{b}=73.5 \AA, \mathrm{c}=94.8 \AA$ in the $\mathrm{P} 41212$ space group, and the $\mathrm{Nb} 2.22$ crystal diffracted to $2.70 \AA$ with $\alpha=\beta=\gamma$ $=90^{\circ}, \mathrm{a}=\mathrm{b}=80.5 \AA, \mathrm{c}=107.7 \AA$, in the $\mathrm{P} 42$ space group.

\section{Structure Determination}

Data were processed with XDS package (24). The structures of Clec4F nanobodies were determined via molecular replacement using Phaser implemented in the Phenix package using nanobody from 5IMM as search model (25). The model was manually improved with the COOT program (26) and refinement was further done using Phenix (25). The interaction interface was calculated by PDB PISA. Figures were generated from PyMOL program. Data collection and refinement statistics are summarized in Supplementary Table 1. Crystallographic coordinates and structure factors were deposited to the Protein 


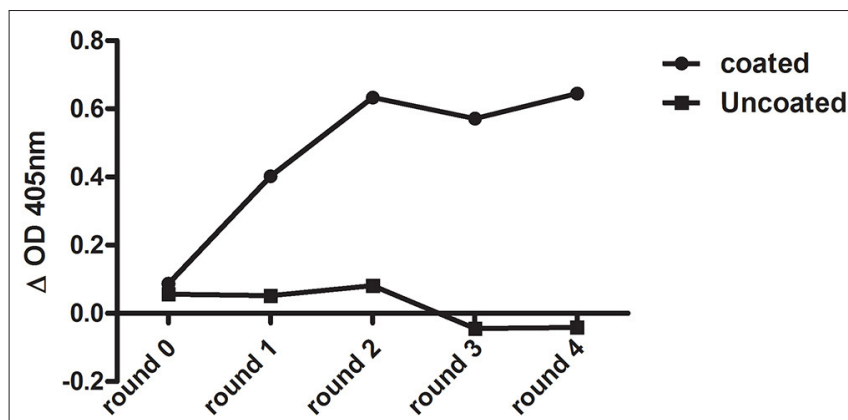

FIGURE 1 | Enrichment of Clec4F-specific nanobody after each round of bio-panning as measured by phage ELISA. Wells were coated with either $1 \mu \mathrm{g} / \mathrm{ml}$ mouse Clec4F recombinant protein (coated), or only with PBS (uncoated). Output phages before panning (round 0 ) and after 4 rounds of biopanning were used as ligands in an ELISA.

Data Bank with access codes 7DJX and 7DJY for Nb1.46 and $\mathrm{Nb} 2.22$.

\section{Statistical Analysis}

Statistical analyses were conducted using the one-way ANOVA assuming unequal variances. Prism 6.0 (GraphPad software) was used for statistical analyses and graph creation. $P$-values $\leq 0.001$ were considered significant.

\section{RESULTS}

\section{Mouse Clec4F Nanobody Generation}

After six rounds of immunization with Clec4F recombinant protein, total mRNA from peripheral blood lymphocyte cells (PBLs) was isolated, then the cDNA was generated using RTPCR. The VHH library with a size of $7.27 \times 10^{7}$ individual clones was expressed on M13 phages and panned against recombinant Clec4F protein coated in microplate wells. In total, four rounds of phage panning were performed. After the third round of panning, antigen-recognizing phages were enriched by a factor of $4.4 \times 10^{4}$. The antigen-specific phages were confirmed in a phage enrichment ELISA against micro-plate coated Clec4F (Figure 1). Periplasmic extracts were generated of 94 colonies from the 2 nd and 47 colonies from the 3 rd round of panning, respectively. When these extracts were tested in ELISA against recombinant mouse $\mathrm{Clec} 4 \mathrm{~F}$ protein, based on the specific signal vs. background signal ratio, 102 out of the $141 \mathrm{Nb}$ clones demonstrated specific antigen binding and were sequenced (Table 1).

Within the $\mathrm{VHH}$, there are three complementarity determining regions (CDRs) or antigen-binding loops. The $3 \mathrm{CDR}$ regions define the unique binding specificity to the antigen epitope, whereby the length and sequence variability of these loops determine the antigen binding affinity. The CDR regions are separated by relatively invariant regions to support the CDRs, called framework regions. Among 102 positive clones, 24 distinct $\mathrm{VHH}$ fingerprints were identified according to the IMGT unique numbering system, belonging to 13 different groups based on their unique CDR3 regions.
TABLE 1 | PE-ELISA of individual antigen-binding Clec4F nanobodies from panning of round 2 and 3 .

\begin{tabular}{lccc}
\hline Specific signal vs. background signal & Round 2 & Round 3 & Total \\
\hline >-fold & 69 & 33 & 102 \\
2-3-fold & 7 & 6 & 13 \\
$<$-fold & 18 & 8 & 26 \\
Total colonies & 94 & 47 & 141
\end{tabular}

The number of colonies are shown with respect to the specific signal generated during the assay. Colonies with the best signal strength difference vs. background signals were DNA sequenced.

The other groups not only showed a unique CDR3 but also unique CDR1 and CDR2 sequences. Representative clones were selected from each group, differing in a few framework region amino acids (Figure 2). In total 23 nanobodies were successfully recloned to the pHEN6c vector for expression with a his-tag at the carboxy-terminus.

\section{Production and Purification of $\mathrm{mClec4F}$ Nanobodies}

WK6 E. coli cells were transformed with the plasmid constructs after which the periplasmic expression of Clec4F nanobodies was performed. All the nanobodies were produced in $400 \mathrm{ml}$ as an initial trial and purified by IMAC and buffer exchanged by dialysis (Supplementary Table 2). The yield of nanobodies varied from $1.1 \mathrm{mg}$ to $16 \mathrm{mg}$ per liter. Nanobodies Nb7.24, $\mathrm{Nb} 4.39, \mathrm{Nb} 11.63$, and Nb6.39 were expressing poorly, therefore we excluded them from later experiments.

The SDS-PAGE of nanobodies Nb10.16, Nb6.19, Nb11.20, Nb10.21, Nb11.55, Nb10.66, Nb14.70, Nb12.75, Nb6.3, Nb6.4, Nb13.12, Nb9.15, Nb11.16, Nb10.17, Nb6.18, Nb2.22, Nb8.37 in elution buffer after IMAC purification and dialysis showed high purity and the characteristic size of nanobodies around $14 \mathrm{kD}$ (Figure 3). The preparations of nanobodies Nb1.46 and Nb3.60 revealed presence of dimers (Figures 3C,D). This was expected for $\mathrm{Nb} 1.46$ as it contained three cysteines, two of which form the conserved disulphide bond and an extra cysteine at position 52 (IMGT numbering) that could form an interdomain disulfide bond. The dimerization of Nb3.60 was unexpected and might be provoked by the short CDR3 whereby the FR4 of two nanobodies are swapped during folding.

\section{The Binding Capacity of the Nanobodies to Clec4F Determined by Surface Plasmon Resonance}

Binding kinetics of nanobodies targeting Clec4F were determined using surface plasmon resonance (SPR). The kinetic interaction between $\mathrm{Clec} 4 \mathrm{~F}$ nanobodies and $\mathrm{Clec} 4 \mathrm{~F}$ yielded a picomolar equilibrium dissociation constant $\left(\mathrm{K}_{\mathrm{D}}\right)$ for most nanobodies. The comparison of the $k_{o n}, k_{o f f}$, and $\mathrm{K}_{\mathrm{D}}$ values of Clec $4 \mathrm{~F}$ nanobodies revealed that 12 nanobodies were found to display excellent offrates from Clec4F (Table 2). The corresponding $k_{\text {off }}$ values of nanobodies from group 2, 6, 10, and 11 were superior compared to the others. Furthermore, the difficult elution and regeneration 


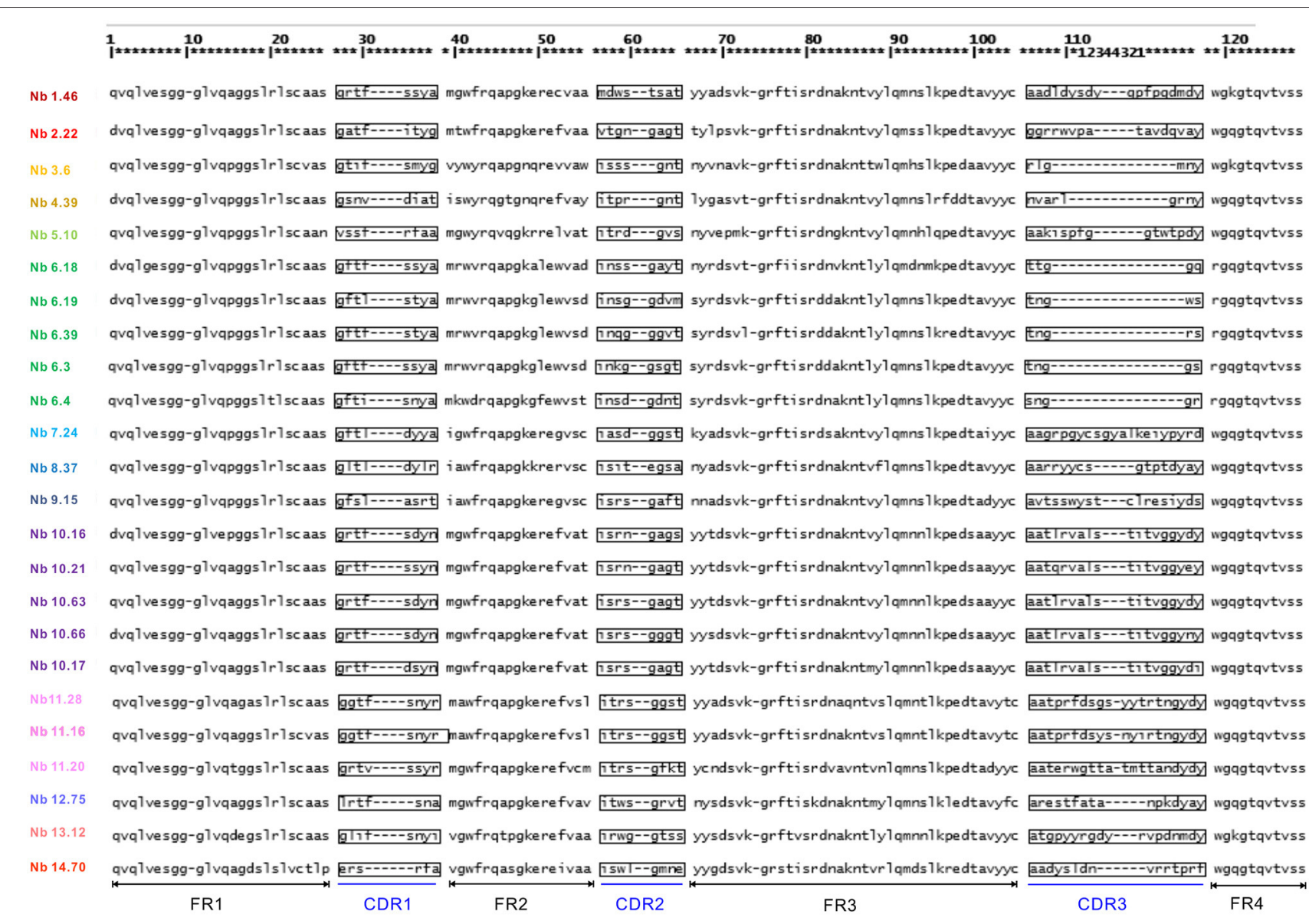

FIGURE 2 | Amino acid sequence of Clec4F nanobodies (numbering according to IMGT). Deduced amino-acid sequences of different nanobodies, consisting of complementarities determining regions (CDR) sequences alternated with structural framework region (FR) sequences.

of $\mathrm{Nb} 1.46,12.75$, and 5.10 from the Clec4F conjugated SRP chip may also suggest they have a good affinity. For the dimerized $\mathrm{Nb} 1.46$ this suggested that at least one entity was active in antigen binding.

\section{Clec4F Nanobodies' Affinity and Epitope Binning}

Clec4F nanobodies from groups $2,3,6,7,8,9,10,11,13$, and 14 were selected for epitope binning using SPR. Since it was very difficult to completely remove $\mathrm{Nb} 1.46, \mathrm{Nb} 5.10$, and $\mathrm{Nb} 12.75$ after the regeneration step, these nanobodies were excluded from the epitope binning experiment. The Clec4F protein was immobilized on a chip and competitive binding between the nanobodies was analyzed by running the nanobodies subsequently over the chip. The first $\mathrm{Nb}$ was flown over the sensor using a concentration of $50 \mathrm{nM}$, which is $10-20$-fold of the $\mathrm{Nb} \mathrm{KD}$ to saturate its epitope. After the 1 st $\mathrm{Nb}$ binding reached equilibrium, a mix of the 1 st $\mathrm{Nb}$ and a $2 \mathrm{nd} \mathrm{Nb}$ was injected to evaluate whether this $2 \mathrm{nd} \mathrm{Nb}$ caused extra binding resonance units (RU), indicating that these nanobodies bind to independent epitopes on Clec4F. As an example, the $\mathrm{Nb} 9.15$ and $\mathrm{Nb} 2.22$ did not affect each other's binding, which demonstrated that they recognize different epitopes since RU levels doubled upon binding of the second nanobody (Figures 4A,B). Next, all the possible combinations of $\mathrm{Nb}$ pairs within the different groups were tested. For example, Nb6.3, Nb6.4, or Nb6.18 did not cause an extra RU upon the binding of Nb6.39, indicating that all members from group 6 bind to the same epitope (Figure 4C). These results corresponded with the similar sequence alignment in CDR3. Finally, the interaction studies between the representative nanobodies from each group are summarized in Supplementary Table 3. Briefly, the SPR study revealed that 4 different epitope groups existed among $\mathrm{Nb}$ sequence groups $2,3,6,7,8,9,10,11,13,14$, which are graphically depicted in Figure 4D.

\section{In vivo Biodistribution of Clec4F Nanobodies}

Clec4F nanobodies that bind non-competitively to Clec4F were purified by size exclusion chromatography and retested for affinity and purity (Figures 5A,B). Four selected nanobodies (Nb3.3, Nb10.16, Nb2.22, and Nb8.37) which exhibited the highest affinity within their epitope group were labeled with ${ }^{99 \mathrm{~m}}$ Technetium $\left({ }^{99 \mathrm{~m}} \mathrm{Tc}\right)$ via their his-tag through tricarbonyl 

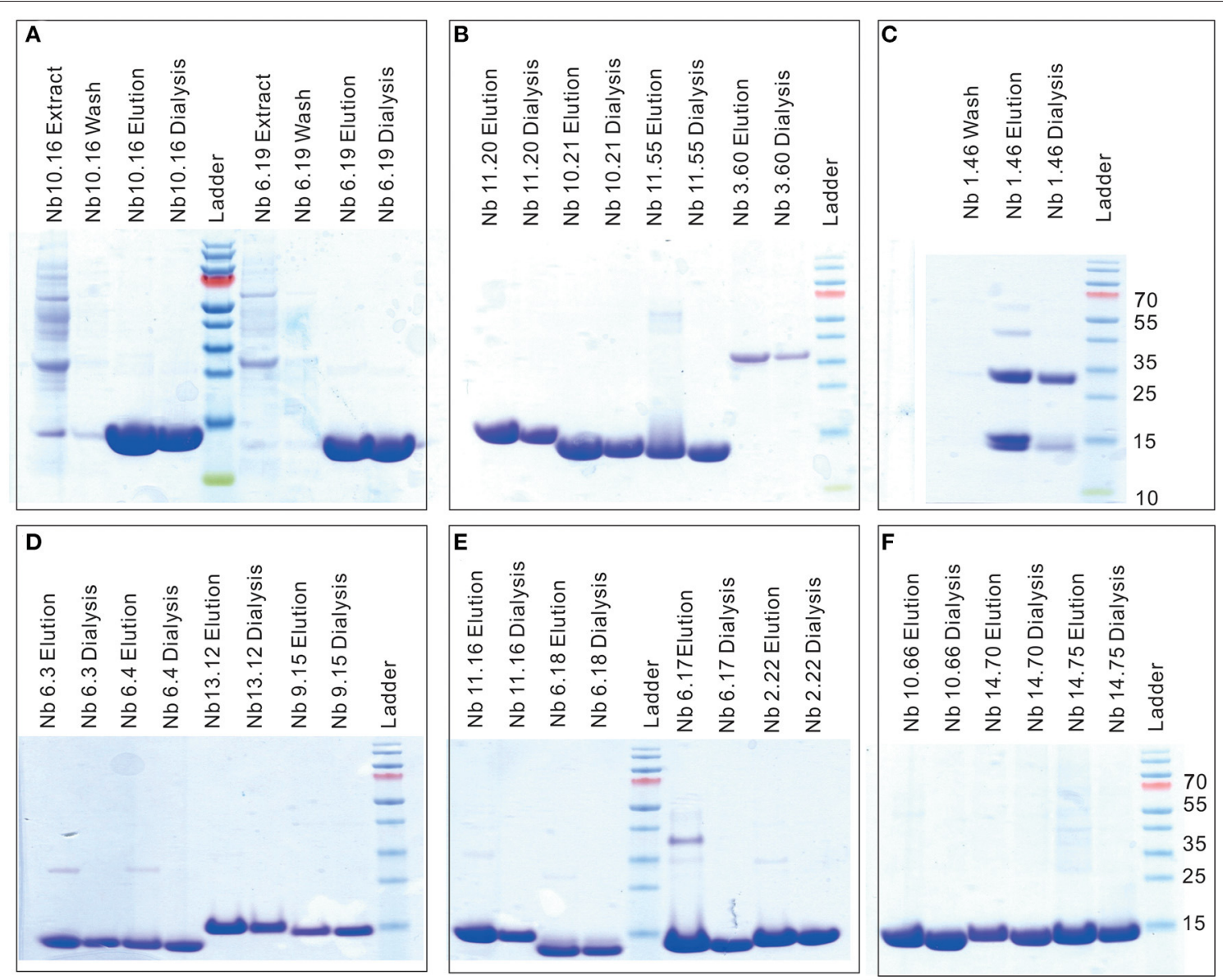

FIGURE 3 I SDS-PAGE to confirm the purity and size of Clec4F nanobodies. Ten percentage SDS-PAGE revealed the presence of (A) Nb10.16, Nb6.19 (B) Nb11.20, $\mathrm{Nb10.21,} \mathrm{Nb11.55,} \mathrm{Nb3.60} \mathrm{(C)} \mathrm{Nb1.46} \mathrm{(D)} \mathrm{Nb6.3,} \mathrm{Nb6.4,} \mathrm{Nb13.12,} \mathrm{Nb9.15} \mathrm{(E)} \mathrm{Nb11.16,} \mathrm{Nb6.18,} \mathrm{Nb6.17,} \mathrm{Nb2.22} \mathrm{(F)} \mathrm{Nb10.66,} \mathrm{Nb14.70} \mathrm{and} \mathrm{Nb14.75,} \mathrm{in}$ (periplasmic) extract, wash flow through (10 mM imidazole-PBS), elution buffer (500 mM imidazole-PBS) and Nb after dialysis (PBS). It showed high purity and a characteristic $\mathrm{Nb}$ size of around $14 \mathrm{kD}$; Nb1.46 and Nb3.60 show dimerized material. M lane: relative molecular mass marker (kDa).

chemistry, purified and filtered. After ${ }^{99 \mathrm{~m}}$ Tc-radiolabeling and subsequent purification steps, labeling efficiencies, reflecting the amount of the added ${ }^{99 \mathrm{~m}} \mathrm{Tc}$ that ended up coupled to the filtered nanobodies, ranged between 50 and $70 \%$ for the various nanobody preparations. Radiochemical purities, reflecting non-free radioactivity coupled to the nanobody in the final preparation, were at least $99 \%$ for all nanobodies. These radiolabeled nanobodies were injected intravenously, followed by dissection of the different organs and measurement of the radioactivity in each organ, to test whether these ${ }^{99 \mathrm{~m}} \mathrm{Tc}-\mathrm{Nbs}$ could target Clec4F positive tissue (liver) in the naive mouse in vivo. The non-targeting control NbBCII10 showed only a significant signal in the kidneys, reflecting $\mathrm{Nb}$ retention in the kidneys after filtration. With the exception of $\mathrm{Nb8.37}$, which showed a significant radioactive signal in blood and other tissues, the other Clec4F nanobodies showed a similar biodistribution pattern: specific uptake was detected in the liver, but not in any of the other organs (Figure $5 \mathrm{C}$ ). ${ }^{99 \mathrm{~m}} \mathrm{Tc}-\mathrm{Nb} 2.22$ with a relative high in vitro affinity for the recombinant target $(0.3 \mathrm{nM})$ also demonstrated superior accumulation in the liver $(p<0.05)$. So, based on these biochemical properties and specifically on the liver targeting properties, $\mathrm{Nb} 2.22$ targeting a unique epitope was selected as lead Clec4F nanobody for future KC imaging studies.

\section{Structural Characterization of $\mathrm{Nb1.46}$ and $\mathrm{Nb2.22}$}

To investigate the diversity of the nanobodies' epitopes and to allow to in the future further optimize them based on rational design, we determined the crystallographic structure of these nanobodies. We tried to obtain crystals from several of the antiClec4F nanobodies. Unfortunately, we could only obtain crystals for $\mathrm{Nb} 1.46$ and $\mathrm{Nb} 2.22$. Nb1.46 has an unpaired cysteine in FR2, and a lysine in FR4 which is unusual for nanobodies. It may explain that $\mathrm{Nb} 1.46$ forms dimers (Figure 3) and has a lower affinity as compared to $\mathrm{Nb2.22}$, as detected by ELISA (Figure 5A). The $\mathrm{Nb} 1.46$ and $\mathrm{Nb} 2.22$ structures were determined through molecular replacement using a Nb119, which is a $\mathrm{Nb}$ with specificity against Vsig4, another KC marker, as a template to a resolution of 1.98 and 2.70 , respectively (27). The overall structure of $\mathrm{Nb} 1.46$ and $\mathrm{Nb} 2.22$ contains $10 \beta$-strands as the 
TABLE 2 | Kinetic rate and equilibrium binding constants of the Clec4F nanobodies, measured using surface plasmon resonance on immobilized recombinant mouse Clec4F protein.

\begin{tabular}{|c|c|c|c|c|c|c|c|}
\hline No & Name & Round & Group & $k_{\text {on }}\left(M^{-1} s^{-1}\right)$ & $k_{\text {off }}\left(s^{-1}\right)$ & $K_{D}(M)$ & $\mathrm{nM}$ \\
\hline 1 & $\mathrm{Nb} 1.46$ & $\mathrm{R} 2-46$ & G1 & \multicolumn{4}{|c|}{ The regeneration is not feasible, not measured. } \\
\hline 2 & $\mathrm{Nb} 2.22$ & R3-22 & G2 & $8.242 E+6$ & 0.002213 & 2.685E-10 & 0.26 \\
\hline 3 & Nb3.60 & $\mathrm{R} 2-60$ & G3 & $7.687 \mathrm{E}+6$ & 0.004750 & $6.180 \mathrm{E}-10$ & 0.61 \\
\hline 4 & Nb4.39 & $\mathrm{R} 2-39$ & G4 & \multicolumn{4}{|c|}{ Poorly expressed. } \\
\hline 5 & Nb5.10 & R3-10 & G5 & \multicolumn{4}{|c|}{ The regeneration is not feasible, not measured. } \\
\hline 6 & Nb6.3 & R3-3 & G6 & $2.868 \mathrm{E}+6$ & $8.484 \mathrm{E}-4$ & $2.958 \mathrm{E}-10$ & 0.29 \\
\hline 7 & $\mathrm{Nb} 6.4$ & R3-4 & G6 & $1.155 \mathrm{E}+6$ & 0.002314 & 2.003E-9 & 2.00 \\
\hline 8 & Nb6.18 & $\mathrm{R} 3-18$ & G6 & $1.482 E+6$ & $7.559 \mathrm{E}-4$ & 5.1001E-10 & 0.51 \\
\hline 9 & $\mathrm{Nb6.19}$ & $\mathrm{R} 2-19$ & G6 & $1.302 E+6$ & $6.125 \mathrm{E}-4$ & 4.704E-10 & 0.47 \\
\hline 10 & Nb6.39 & R3-39 & G6 & \multicolumn{4}{|c|}{ Poorly expressed. } \\
\hline 11 & $\mathrm{Nb} 7.24$ & $\mathrm{R} 2-24$ & G7 & \multicolumn{4}{|c|}{ Poorly expressed. } \\
\hline 12 & Nb8.37 & R3-37 & G8 & $1.573 E+6$ & 0.001243 & 7.902E-10 & 0.79 \\
\hline 13 & Nb9.15 & R3-15 & G9 & $1.189 E+6$ & 0.03171 & $2.668 \mathrm{E}-8$ & 26 \\
\hline 14 & Nb10.16 & $\mathrm{R} 2-16$ & G10 & $1.576 \mathrm{E}+6$ & $8.252 \mathrm{E}-4$ & 5.237E-10 & 0.52 \\
\hline 15 & Nb10.17 & R3-17 & G10 & $9.082 E+6$ & 0.001584 & $1.744 \mathrm{E}-10$ & 0.17 \\
\hline 16 & Nb10.21 & $\mathrm{R} 2-21$ & G10 & $4.619 E+6$ & 0.002233 & $4.835 \mathrm{E}-10$ & 0.48 \\
\hline 17 & Nb10.66 & $\mathrm{R} 2-66$ & G10 & $4.280 E+6$ & 0.001345 & $3.143 E-10$ & 0.31 \\
\hline 18 & Nb11.16 & R3-16 & G11 & $2.320 \mathrm{E}+7$ & 0.003921 & $1.690 \mathrm{E}-10$ & 0.16 \\
\hline 19 & $\mathrm{Nb} 11.20$ & $\mathrm{R} 2-20$ & G11 & $3.459 E+6$ & 0.001395 & 4.033E-10 & 0.40 \\
\hline 20 & Nb11.28 & R3-28 & G11 & $5.432 E+6$ & 0.006535 & $1.203 E-9$ & 1.20 \\
\hline 21 & Nb11.55 & $\mathrm{R} 2-55$ & G11 & $4.332 E+6$ & 0.002150 & 4.964E-10 & 0.49 \\
\hline 22 & Nb11.63 & $\mathrm{R} 2-63$ & G11 & \multicolumn{4}{|c|}{ Poorly expressed. } \\
\hline 23 & $\mathrm{Nb} 12.75$ & $\mathrm{R} 2-75$ & G12 & \multicolumn{4}{|c|}{ The regeneration is not feasible, not measured. } \\
\hline 24 & Nb13.12 & R3-12 & G13 & $2.788 \mathrm{E}+6$ & 0.003097 & $1.111 \mathrm{E}-9$ & 1.11 \\
\hline 25 & Nb14.70 & $\mathrm{R} 2-70$ & G14 & $3.061 E+7$ & 0.06062 & $1.980 \mathrm{E}-9$ & 0.19 \\
\hline
\end{tabular}

The affinities $\left(K_{D}\right)$, on-rate $\left(k_{o n}\right)$, off-rate $\left(k_{\text {off }}\right)$ are calculated based on fitting of observed interaction curves of a dilution series of nanobodies.

canonical immunoglobulin fold of $\mathrm{VHH}$ in two antiparallel $\beta$ sheets. The folded VHH fragment forms an extended interface that interacts with the antigen, known as paratope. The Nb1.46 has CDR1 to 3 comprising 8 residues $\left(\mathrm{Glu}^{27}\right.$ to $\mathrm{Ala}^{38}$ ), 8 residues $\left(\mathrm{Met}^{51}\right.$ to $\mathrm{Thr}^{58}$ ) and 16 residues (Asp ${ }^{99}$ to $\mathrm{Tyr}^{114}$ ), respectively. The $\mathrm{Nb} 2.22$ has CDR1 to 3 comprising 8 residues $\left(\mathrm{Glu}^{27}\right.$ to $\mathrm{Tyr}^{38}$ ), 8 residues $\left(\mathrm{Val}^{51}\right.$ to $\mathrm{Thr}^{58}$ ) and 14 residues ( $\mathrm{Arg}^{99}$ to $\mathrm{Tyr}^{112}$ ), respectively (Figure 6A). Nb1.46 and $\mathrm{Nb} 2.22$ have 8 residues in CDR1 and among them there are four residues that are different.

As shown in the structure alignment, the CDR1 of Nb1.46 and $\mathrm{Nb} 2.22$ almost coincide (Figure 6B). However, Nb1.46 and $\mathrm{Nb} 2.22$ have 8 completely different residues in the CDR2, resulting in the CDR2 loop bending into different orientations since CDR2 of $\mathrm{Nb} 2.22$ has residues Gly ${ }^{58}$ and Gly ${ }^{62}$ that make the surface more hydrophobic. Antigen binding is mediated by noncovalent interactions, mainly involving amino acids in the CDRs, mostly in CDR3. Interestingly, the CDR3 of Clec4F nanobodies includes a short $\beta$-strand. $\mathrm{Nb} 2.22$ has a shorter version of the CDR3 loop as compared to $\mathrm{Nb} 1.46$ indicating a completely different pattern of binding with Clec4F. Nb1.46 and Nb2.22 also harbor two cysteines ( $\mathrm{Cys}^{23}$ and $\mathrm{Cys}^{104}$ ) that form the disulfide bridge connecting the two $\beta$-strands to constrain the antigenbinding loops and maintain the flexibility (Figure 6C). However, an interdomain disulphide bond of $\mathrm{Cys}^{52}$ may mediate the dimer formation in the Nb1.46.

\section{DISCUSSION}

The liver performs a wide range of functions, including detoxification, protein synthesis, production of biochemicals necessary for digestion, clearance of macromolecules from the blood and their metabolization (28). The liver KCs play an important role in surveillance, uptake of immune complexes, tumor cells, liposomes, lipid microspheres, iron, immune complexes and other blood-borne particulates and as regulatory and effector cells in immune responses to infectious agents and other conditions challenging liver homeostasis. To optimally dissect the role of the various cell types in the liver, it is a crucial asset to identify the resident KCs from other myeloid cells such as circulating monocytes and different types of DCs or recently immigrated macrophages within populations of liver cells after isolation and under inflammatory conditions.

Clec4F has a carbohydrate-recognition domain (CRD) that recognizes glycans in a $\mathrm{Ca}^{2+}$-dependent (C-type) manner, an $\mathrm{N}$-terminal cytoplasmic signaling domain, a transmembrane hydrophobic helix, and a heptad neck region, which stabilizes trimer formation. As we have shown in our previous studies, 
A

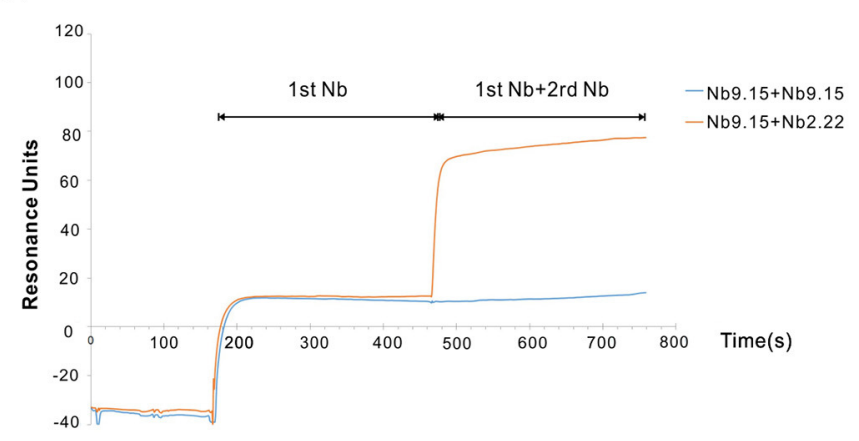

B

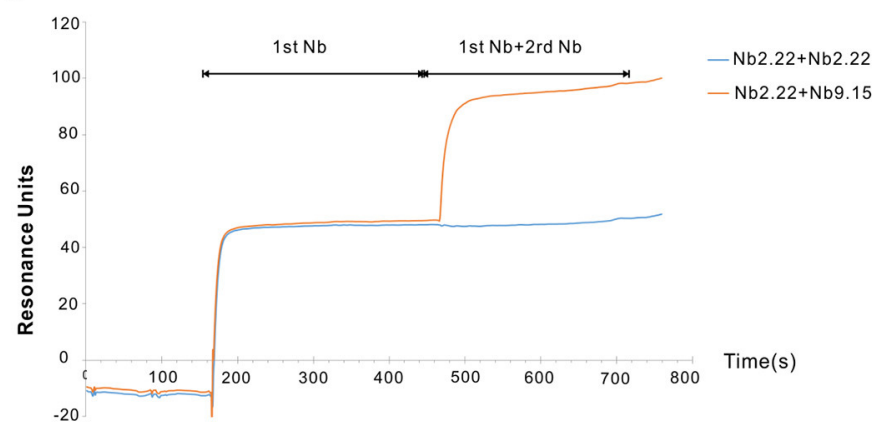

C

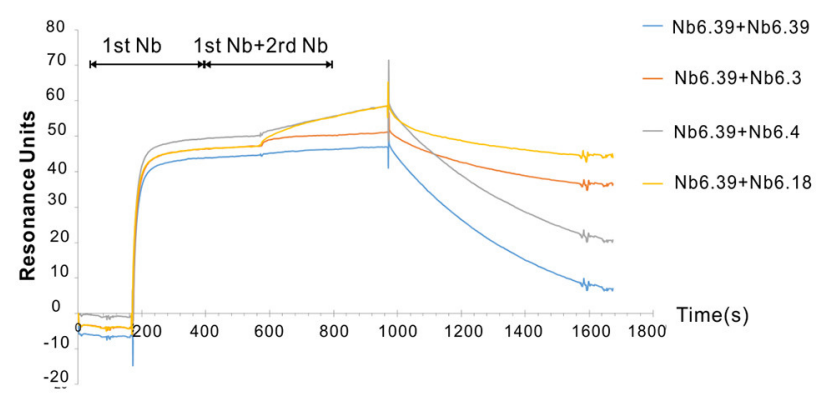

D

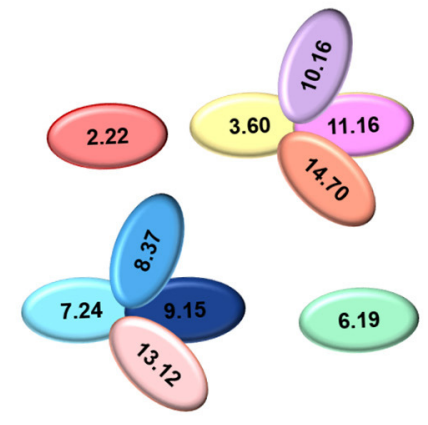

FIGURE 4 | Clec4F nanobodies epitope binning by SPR. Examples of lack of overlap between Nb2.22 (2rd Nb) and Nb9.15(1st Nb) epitopes on Clec4F antigen (A) and vice versa of Nb9.15 (2st Nb) and Nb2.22 (1st Nb) to Clec4F (B), indicating that these bind to different epitopes. (C) The inhibitory effect of nanobodies within group 6. RU refers to resonance units. (D) Summary of Clec4F Nb epitope mappings with different colors indicating different epitope groups. The nanobodies are named according to different groups in CDR3.

Clec4F is only expressed by Kupffer cells and is absent in infiltrating monocytes. Therefore, $\mathrm{Clec} 4 \mathrm{~F}$ can be used as a specific Kupffer cell marker to study the roles of distinct populations present in the liver (29). The loss of Clec $4 \mathrm{~F}^{+}$Kupffer cells was shown to be a sign of extensive liver damage upon CrimeanCongo hemorrhagic fever virus (CCHFV) infections in mice (30). Clec4F has also been shown to be necessary for the clearance of desialylated platelets due to the recognition of exposed galactose epitopes (5). Moreover, Clec4F was reported to be involved in the Listeria monocytogenes infection in mouse liver (9).

A few years ago, we have reported that molecular imaging with Kupffer cell-targeting nanobodies can be used for diagnosis and prognosis in mouse models of liver pathogenesis, whereby we used nanobodies targeting two distinct receptors on Kupffer cells, namely Vsig4 and Clec4F (20). The nanobodies against these two targets were used as tools for imaging therein, but their generation was not the focus of that publication. Meanwhile, we have also reported the structure of a truncated trimeric mouse Clec4F containing the CRD and part of the heptad neck domain. The Clec4F trimeric structure reveals two unique conserved calcium-binding sites, which differ from that of Langerin and other C-type lectins, which may contribute to the unique recognition pattern of Clec4F (11). Having solved the structure of Clec4F, has renewed the interest in the anti-Clec4F nanobodies and we have now also solved the structure of these anti-Clec4F nanobodies. In the current manuscript, we provide more detailed information on the initial generation and characterization of the nanobodies, including nanobody sequence data, epitope binning data and initial in vivo biodistribution data of various Clec4F nanobodies, which were not published before, and have combined these with our new findings on the crystal structure of $\mathrm{Nb} 1.46$ and $\mathrm{Nb} 2.22$.

Clec4F-specific nanobodies were obtained by direct cloning of the $\mathrm{VHH}$ genes from B-cells obtained from an immunized alpaca, and selection via phage-display and bio-panning. The Clec4F nanobodies showed good diversity, as reflected in the number of different sequences obtained after two and three rounds of panning. Twenty-five nanobodies were categorized into 14 groups based on sequence identity in their CDR3, which is the region of the $\mathrm{VHH}$ with the largest variability. In these $25 \mathrm{Clec} 4 \mathrm{~F}$ nanobodies the length of CDR3 is in the range 621AA, whereby the nanobodies from group 6 have the shortest CDR3. Within each group these nanobodies have one or more mutations in other regions such as CDR1 and CDR2, which may also be related to their differences in binding capacity and expression levels. These 25 nanobodies were expressed in $E$. coli and purified from $400 \mathrm{~mL}$ cultures in TB medium, with variable yields. These nanobodies showed a limited variation in molecular weight, as shown by using SDS PAGE analysis. The $K_{D}$ values of these nanobodies are in the range from 
A

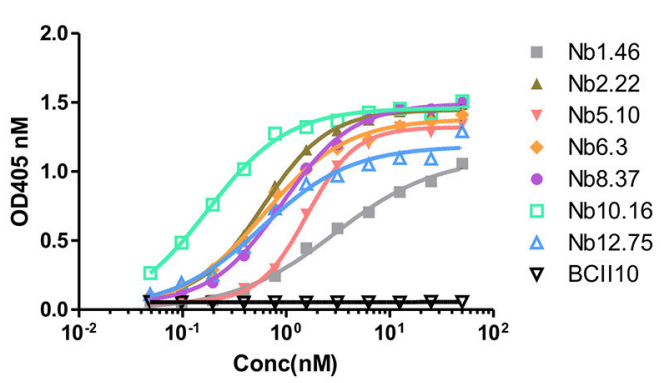

B

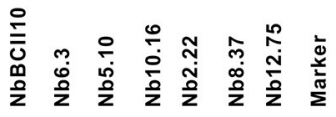

C

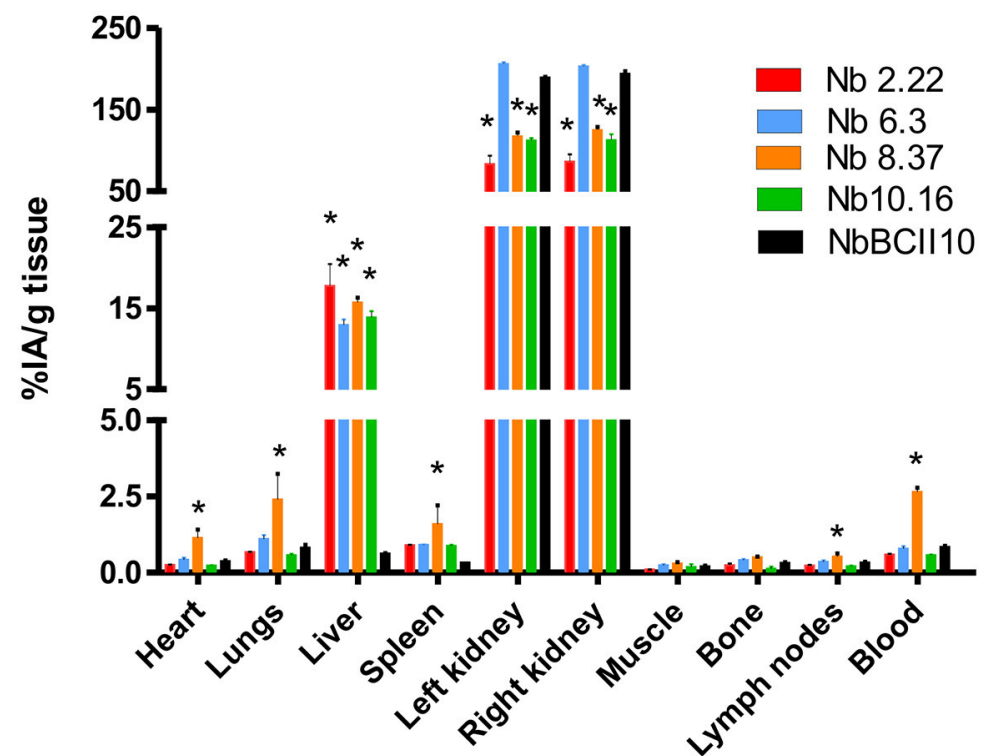

FIGURE 5 | Binding characteristics of Clec4F nanobodies. (A) Binding of increasing concentrations of Clec4F nanobodies was tested on recombinant Clec4F protein using ELISA. (B) SDS-PAGE confirmed the purity and size of selected Clec4F nanobodies after purification by size exclusion chromatography. (C) Biodistribution of radiolabeled Clec4F Nanobodies in naive mice. Four distinct ${ }^{99 \mathrm{~m}} \mathrm{Tc}-\mathrm{Clec} 4 \mathrm{~F}$ nanobodies and ${ }^{99 \mathrm{~m}} \mathrm{Tc}-\mathrm{NbBCll10}$ control Nb were injected in naive mice (mean of 3 mice \pm SEM). Radioactive content of each organ was measured in a $\gamma$-counter and expressed for each $\mathrm{Nb}$ as a percentage of injected radioactivity per gram of tissue $(\% \mathrm{IA} / \mathrm{g}),{ }^{*} P<0.001$ vs. ${ }^{99 \mathrm{~m}} \mathrm{Tc}-\mathrm{NbBCll} 10$ control $\mathrm{Nb}$.

0.19 to $26 \mathrm{nM}$. Using a competitive assay via SPR we could determine that $\mathrm{Nb} 2.22$ from Group 2 and $\mathrm{Nb} 6.19$ from Group 6 bind to two different epitopes of Clec4F. The nanobodies of group 3,10,11, and 14 seem to inhibit each other's binding, suggesting they bind to a common epitope. The nanobodies of group 7, 8, 9 and 13 form a fourth epitope group. Therefore, we can distinguish at least four main epitope groups among these mouse Clec4F-binding nanobodies. Based on in vivo liver biodistribution in naive mice, we have observed that $\mathrm{Nb}$ 2.22, 3.3, 8.37 , and $\mathrm{Nb} 10.16$ effectively target the liver. Of note, additional validation data of the $\mathrm{Nb} 2.22$, which was selected as lead $\mathrm{Clec} 4 \mathrm{~F}$ nanobody for KC imaging studies, have been published before (20). In particular, we have shown using Clec4F-DTR depleter mice, allowing for the specific depletion of KCs when treated with DT, that accumulation of the ${ }^{99 \mathrm{~m}} \mathrm{Tc}$-labeled $\mathrm{Nb} 2.22$ is not observed in the liver of KC-depleted mice. Similarly, the liver accumulation of ${ }^{99 \mathrm{~m}} \mathrm{Tc}-\mathrm{Nb} 2.22$ also disappears in liver after chlodronate liposome depletion of phagocytes. These data confirm that the in vivo imaging signal in the liver is restricted to KCs. Moreover, immunofluorescence microscopy using $\mathrm{Nb}$ 2.22 on liver sections of naive mice has revealed that Clec4F costains F4/80-expressing cells. Finally, in flow cytometry analysis on liver single cell suspensions, $\mathrm{Nb} 2.22$ stains CD11b ${ }^{\text {int }} \mathrm{F} 4 / 80^{+}$ $\mathrm{KCs}$, but not $\mathrm{F} 480^{\text {low }} \mathrm{CD} 11 \mathrm{~b}^{\text {high }}$ inflammatory monocytes or Ly6G-expressing polymorphonuclear cells (20).

To have a basis for better understanding of the mode of action of these nanobodies and the different affinities, the crystal structures of Nb1.46 and Nb2.22 were obtained. The Nb1.46 has an unpaired cysteine in the FR2. Nb2.22 has a conserved intradomain disulfide bond giving rise to a disulfide bridge joining the CDR1 and CDR3, as is often found in other conventional VHH domains. Such bridge would turn the loop into a more rigid structure, a relevant feature when considering its thermodynamic consequences. Furthermore, the polar amino acid substitutions 


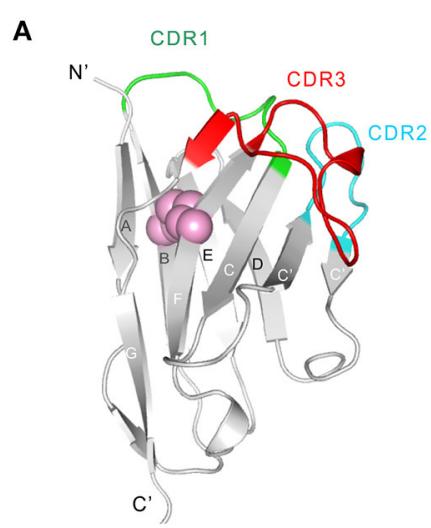

Nb1.46

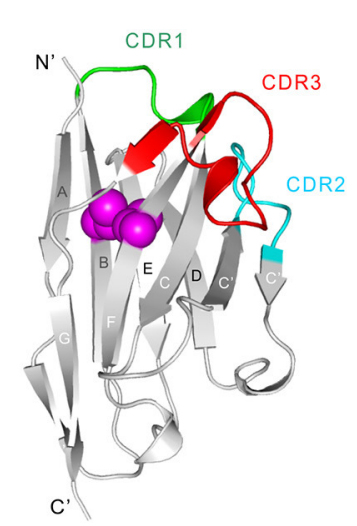

Nb2.22
B

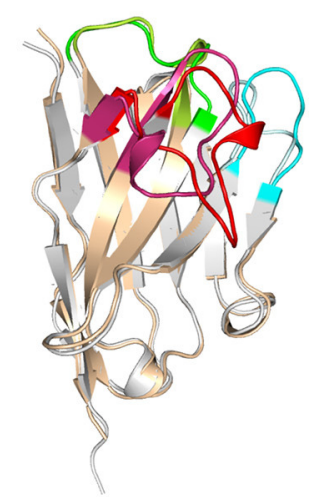

Alignment

\section{C}
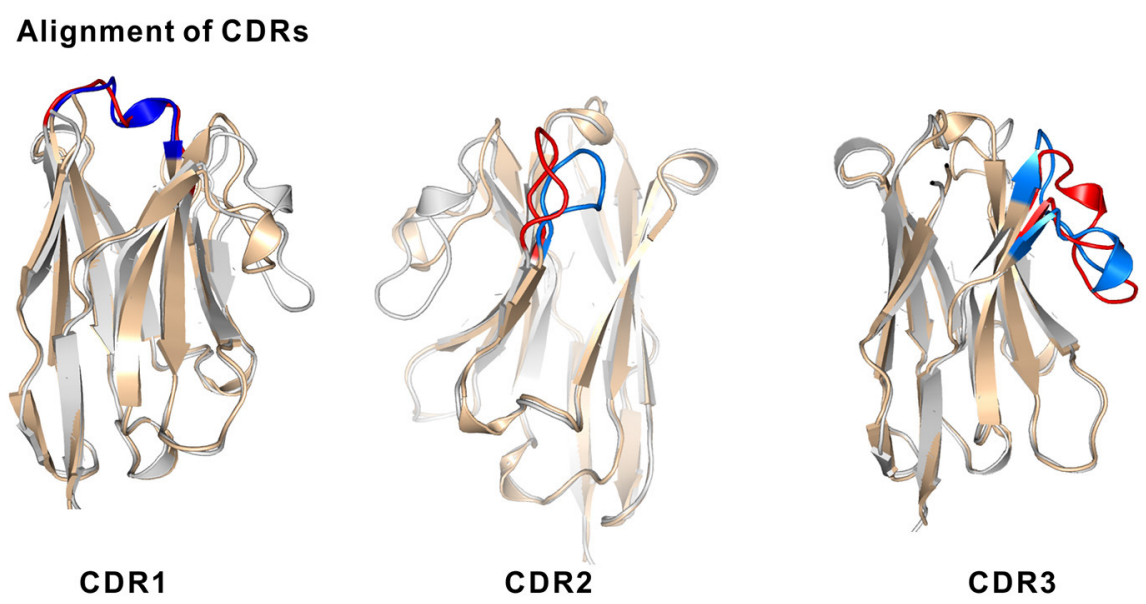

FIGURE 6 | Structural basis of Nb1.46 and Nb2.22. (A) The cartoon view of the Nb1.46 and Nb2.22, the CDR1, CDR2, CDR3 are shown in the color green, cyan and red, respectively, the cysteine involved in disulfide bond formation are denoted as pink sphere for $\mathrm{Nb} 1.46$ and violet sphere for $\mathrm{Nb2}$.22. (B) The alignment of $\mathrm{Nb} 1.46$ to

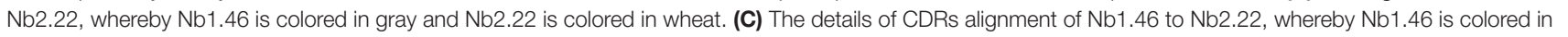
gray and its CDR in red, whereas Nb2.22 is colored in wheat and its CDR in blue.

of $\mathrm{Glu}^{49}$ and $\mathrm{Arg}^{50}$ increase the hydrophilicity of the $\mathrm{VHH}$ surface. Also residues $\mathrm{Gly}^{58}$ and Gly ${ }^{62}$ in CDR2 make the surface more hydrophobic, resulting in the CDR2 loop bending into a different orientation as compared to that one of Nb1.46. We also notice that the substitutions at positions $\mathrm{Phe}^{42}$ and $\mathrm{Cys}^{52}(\mathrm{Nb} 1.46)$ or $\mathrm{Phe}^{52}(\mathrm{Nb} 2.22)$ cause a net shift of the bulky hydrophobic groups toward the center of the sheet and the CDR3 loop folds over to these residues to make them solvent inaccessible. CDR3 of Clec4F nanobodies form a large protruding loop and together with CDR1 and CDR2, the antigen binding surface is as large as that of a scFv. Therefore, the antigen binding capacity of $\mathrm{VHH}$ and $\mathrm{scFv}$ is often in the same range.

Clec4F is a potential candidate biomarker restricted to the liver microenvironment. In the present study, we generated and evaluated anti-Clec4F nanobodies which are small binding moieties, that provide high affinity, but smaller size as compared to complete antibodies. These nanobodies lack the Fc region, and are therefore unable to recruit Fc-mediated effector activity. The superior penetration potential of nanobodies due to their smaller size, combined with high affinity target binding and fast clearance from the circulation, represents an ideal basis for imaging purposes $(21,31)$. By blocking the targets, nanobodies have been reported as efficient enzyme inhibitors for multiple usages (32) such as inhibiting the HIV-1 replication by blocking the Rev protein as an intrabody (33). The ability of nanobodies to recognize different antigenic regions on a protein such as cryptic, hidden or conformational target epitopes offers an added value for their applications. Finally, nanobodies can be easily expressed in E. coli where they can be economically produced as soluble and non-aggregating recombinant proteins in high yields (34).

In conclusion, our study has revealed a group of nanobodies that recognize distinct epitopes on Clec4F. Two of the Clec4F nanobodies were crystallized and the structures were determined through molecular replacement using a Nb119 as a template to 
a resolution of 1.98 and 2.70 , respectively. These two nanobodies share many structural features, but the Nb1.46 and Nb2.22 CDR2 loops bend into different orientations, related to the 8 completely different residues in the CDR2. The Clec4F nanobodies presented here enable further investigation of $\mathrm{Clec} 4 \mathrm{~F}$ biological function using antibody-related methods such as flow cytometry, immunostaining, antigen purification and molecular imaging, as well as offer potential for developing specific isolation of KCs in vitro by $\mathrm{Nb}$ based immune affinity methods. Future work on these nanobodies will address their galactose neutralizing capacity on Clec4F. The determined molecular structure presented here will help in developing blocking nanobodies, which may find applications in blocking Clec4F interactions in disease models such as Listeria monocytogenes infection.

\section{DATA AVAILABILITY STATEMENT}

The datasets generated in this study can be found in online repositories. The names of the repository/repositories and accession number(s) can be found below: http://www.wwpdb. org/, 7DJX; http://www.wwpdb.org/, 7DJY.

\section{ETHICS STATEMENT}

All applicable institutional and/or national guidelines for the care and use of animals were followed and all animal experiments were approved by the Ethical Committee for Animal Experiments of the Vrije Universiteit Brussel (Lab Accreditation Number: LA1210220).

\section{REFERENCES}

1. Locati M, Curtale G, Mantovani A. Diversity, mechanisms, and significance of macrophage plasticity. Annu Rev Pathol Mech Dis. (2020) 15:123-47. doi: 10.1146/annurev-pathmechdis-012418-012718

2. Chambers ES, Vukmanovic-Stejic M. Skin barrier immunity and ageing. Immunology. (2020) 160:116-25. doi: 10.1111/imm.13152

3. Ardain A, Marakalala MJ, Leslie A. Tissue-resident innate immunity in the lung. Immunology. (2020) 159:245-56. doi: 10.1111/imm.13143

4. Dou L, Shi X, He X, Gao Y. Macrophage phenotype and function in liver disorder. Front Immunol. (2020) 10:1-11. doi: 10.3389/fimmu.2019.03112

5. Li Y, Fu J, Ling Y, Yago T, McDaniel JM, Song J, et al. Sialylation on O-glycans protects platelets from clearance by liver Kupffer cells. Proc Natl Acad Sci. (2017) 114:8360-5. doi: 10.1073/pnas.1707662114

6. Hajira Basit, Michae L. Tan DRW. Histology, Kupffer Cell. StatPearls, Treasure Island, FL: StatPearls Publishing, LLC (2020).

7. Zheng F, Devoogdt N, Sparkes A, Morias Y, Abels C, Stijlemans B, et al. Monitoring liver macrophages using nanobodies targeting Vsig4: concanavalin A induced acute hepatitis as paradigm. Immunobiology. (2015) 220:200-9. doi: 10.1016/j.imbio.2014.09.018

8. De Groeve K, Deschacht N, De Koninck C, Caveliers V, Lahoutte T, Devoogdt $\mathrm{N}$, et al. Nanobodies as tools for in vivo imaging of specific immune cell types. J Nucl Med. (2010) 51:782-9. doi: 10.2967/jnumed.109.070078

9. Yang C, Chen J, Tsai T, Tsai Y, Tsai C, Liang P, et al. CLEC4F is an inducible C-type lectin in F4/80-positive cells and is involved in alpha-galactosylceramide presentation in liver. PloS ONE. (2013) 8:E65070. doi: 10.1371/journal.pone.0065070

10. Scott CL, Zheng F, De Baetselier P, Martens L, Saeys Y, De Prijck S, et al. Bone marrow-derived monocytes give rise to self-renewing and fully differentiated Kupffer cells. Nat Commun. (2016) 7:10321. doi: 10.1038/ncomms10321

\section{AUTHOR CONTRIBUTIONS}

FZ, SS, SM, ND, JV, GR, and YW designed the experiments. FZ and JZha performed the characterization experiments. YW, ZO, JZho, and XW performed crystallography experiments. YW and FZ analyzed data. FZ and YW wrote the manuscript. SS, JV, SM, and GR made manuscript revisions. All authors contributed to the article and approved the submitted version.

\section{FUNDING}

YW and FZ are partially supported by National Natural Science Foundation of China (No. 31870132, 82072237, 81501527, and $81772745)$. YW is also supported by Shaanxi Province Natural Science Funding (No. 2020-JQ084).

\section{ACKNOWLEDGMENTS}

We thank the staff assistant from Shanghai Synchrotron Radiation Facility BL18U1 and BL19U1 for help with data collection. We thank Cindy Peleman for valuable technical assistance in performing in vivo imaging experiments. We thank Patrick De Baetselier for fruitful discussions and valuable moral support.

\section{SUPPLEMENTARY MATERIAL}

The Supplementary Material for this article can be found online at: https://www.frontiersin.org/articles/10.3389/fimmu. 2021.641819/full\#supplementary-material

11. Ouyang Z, Felix J, Zhou J, Pei Y, Ma B, Hwang PM, et al. Trimeric structure of the mouse Kupffer cell C-type lectin receptor Clec4f. FEBS Lett. (2020) 594:189-98. doi: 10.1002/1873-3468.13565

12. Taylor ME, Snelling T, Smith DF, Drickamer K. Absence of a human ortholog of rodent Kupffer cell galactose-binding receptor encoded by the CLEC4f gene. Glycobiology. (2019) 29:332-45. doi: 10.1093/glycob/ cwyl13

13. Devisscher L, Scott CL, Lefere S, Raevens S, Bogaerts E, Paridaens $A$, et al. Non-alcoholic steatohepatitis induces transient changes within the liver macrophage pool. Cell Immunol. (2017) 322:74-83. doi: 10.1016/j.cellimm.2017.10.006

14. Scott CL, T'Jonck W, Martens L, Todorov H, Sichien D, Soen B, et al. The transcription factor ZEB2 is required to maintain the tissue-specific identities of macrophages. Immunity. (2018) 49:312-25.e5. doi: 10.1016/j.immuni.2018.07.004

15. Godfrey DI, Kronenberg M. Review series Going both ways: immune regulation via CD1d-dependent NKT cells. J Clin Invest. (2004) 114:1379-88. doi: 10.1172/JCI200423594

16. Debie P, Lafont C, Defrise M, Hansen I, van Willigen DM, van Leeuwen FWB, et al. Size and affinity kinetics of nanobodies influence targeting and penetration of solid tumours. J Control Release. (2020) 317:34-42. doi: 10.1016/j.jconrel.2019.11.014

17. Hoey RJ, Eom H, Horn JR. Structure and development of single domain antibodies as modules for therapeutics and diagnostics. Exp Biol Med. (2019) 244:1568-76. doi: 10.1177/1535370219881129

18. Hassanzadeh-Ghassabeh G, Devoogdt N, De Pauw P, Vincke C, Muyldermans S. Nanobodies and their potential applications. Nanomedicine (Lond). (2013) 8:1013-26. doi: 10.2217/nnm.13.86

19. Jovčevska I, Muyldermans S. The therapeutic potential of nanobodies. BioDrugs. (2020) 34:11-26. doi: 10.1007/s40259-019-00392-Z 
20. Zheng F, Sparkes A, de Baetselier P, Schoonooghe S, Stijlemans B, Muyldermans S, et al. Molecular imaging with kupffer celltargeting nanobodies for diagnosis and prognosis in mouse models of liver pathogenesis. Mol Imaging Biol. (2016) 19:1-10. doi: 10.1007/s11307-016-0976-3

21. Zheng F, Perlman H, Matthys P, Wen Y, Lahoutte T, Muyldermans S, et al. Specificity evaluation and disease monitoring in arthritis imaging with complement receptor of the ig superfamily targeting nanobodies. Sci Rep. (2016) 6:e35966. doi: 10.1038/srep35966

22. Movahedi K, Schoonooghe S, Laoui D, Houbracken I, Waelput W, Breckpot $\mathrm{K}$, et al. Nanobody-based targeting of the macrophage mannose receptor for effective in vivo imaging of tumor-associated macrophages. Cancer Res. (2012) 72:4165-77. doi: 10.1158/0008-5472.CAN-11-2994

23. Put S, Schoonooghe S, Devoogdt N, Schurgers E, Avau A, Mitera T, et al. SPECT imaging of joint inflammation with nanobodies targeting the macrophage mannose receptor in a mouse model for rheumatoid arthritis. J Nucl Med. (2013) 54:807-14. doi: 10.2967/jnumed.112.111781

24. Kabsch W. Xds. Acta Crystallogr Sect D Biol Crystallogr. (2010) 66:125-32. doi: 10.1107/S0907444909047337

25. Adams PD, Afonine PV, Bunkóczi G, Chen VB, Davis IW, Echols N, et al. PHENIX: a comprehensive Python-based system for macromolecular structure solution. Acta Crystallogr Sect D Biol Crystallogr. (2010) 66:213-21. doi: 10.1107/S0907444909052925

26. Emsley P, Cowtan K. Coot: model-building tools for molecular graphics. Acta Crystallogr Sect D. (2004) 60:2126-32. doi: 10.1107/S0907444904019158

27. Wen Y, Ouyang Z, Schoonooghe S, Luo S, De Baetselier P, Lu W, Muyldermans S, et al. Structural evaluation of a nanobody targeting complement receptor Vsig4 and its cross reactivity. Immunobiology. (2017) 222:807-13. doi: 10.1016/j.imbio.2016.11.008

28. Jenne CN, Kubes P. Immune surveillance by the liver. Nat Immunol. (2013) 14:996-1006. doi: 10.1038/ni.2691

29. van der Tuin SJL, Li Z, Berbée JFP, Verkouter I, Ringnalda LE, Neele AE, et al. Lipopolysaccharide lowers cholesteryl ester transfer protein by activating
F4/80+Clec4f+Vsig4+Ly6C- Kupffer Cell Subsets. J Am Heart Assoc. (2018) 7:e008105. doi: 10.1161/JAHA.117.008105

30. Lindquist ME, Zeng X, Altamura LA, Daye SP, Delp KL, Blancett C, et al. Exploring crimean-congo hemorrhagic fever virus-induced hepatic injury using antibody-mediated type I interferon blockade in mice. J Virol. (2018) 92:e01083. doi: 10.1128/JVI.01083-18

31. Demine S, Garcia Ribeiro R, Thevenet J, Marselli L, Marchetti P, Pattou F, et al. A nanobody-based nuclear imaging tracer targeting dipeptidyl peptidase 6 to determine the mass of human beta cell grafts in mice. Diabetologia. (2020) 63:825-36. doi: 10.1007/s00125-019-05068-5

32. Lauwereys M, Arbabi Ghahroudi M, Desmyter A, Kinne J, Hölzer W, De Genst E, et al. Potent enzyme inhibitors derived from dromedary heavy-chain antibodies. EMBO J. (1998) 17:3512-20. doi: 10.1093/emboj/17.13.3512

33. Ahangarzadeh S, Payandeh Z, Arezumand R, Shahzamani K, Yarian F, Alibakhshi A. An update on antiviral antibody-based biopharmaceuticals. Int Immunopharmacol. (2020) 86:e106760 doi: 10.1016/j.intimp.2020. 106760

34. Arbabi-Ghahroudi M, Tanha J, MacKenzie R. Prokaryotic expression of antibodies. Cancer Metastasis Rev. (2005) 24:501-19. doi: 10.1007/s10555-005-6193-1

Conflict of Interest: The authors declare that the research was conducted in the absence of any commercial or financial relationships that could be construed as a potential conflict of interest.

Copyright (c) 2021 Zheng, Zhou, Ouyang, Zhang, Wang, Muyldermans, Van Ginderachter, Devoogdt, Wen, Schoonooghe and Raes. This is an open-access article distributed under the terms of the Creative Commons Attribution License (CC BY). The use, distribution or reproduction in other forums is permitted, provided the original author(s) and the copyright owner(s) are credited and that the original publication in this journal is cited, in accordance with accepted academic practice. No use, distribution or reproduction is permitted which does not comply with these terms. 